\title{
Thickening carbon dioxide with amphiphilic block copolymer
}

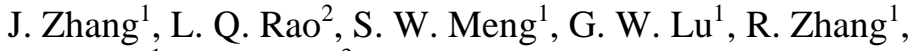 \\ W. S. $\mathrm{Yu}^{1} \&$ Q. Yang \\ ${ }^{1}$ College of Science and College of Petroleum Engineering, \\ China University of Petroleum, China \\ ${ }^{2}$ Huanding Energy Services, China \\ ${ }^{3}$ Tuha division, China Petroleum Logging Co. Ltd., China
}

\begin{abstract}
Liquid carbon dioxide has been previously used successfully in the petroleum industry, such as in enhanced oil recovery (EOR) and fracturing. The application of liquid carbon dioxide in wells not only effectively enhances the oil recovery but also considerably reduces greenhouse gas emissions. The application shortcomings of carbon dioxide is its extremely low viscosity. In 2000, Huang et al. found that amphiphilic fluorinated acrylate (PHFDA)/styrene (PSt)/ sulfonated styrene $(\mathrm{S})$ copolymers can enhance the viscosity of carbon dioxide over 100 -fold at concentrations of about $5 \mathrm{wt} \%$. To further improve the efficiency, optimize the proportion of PHFDA, PSt and S. The simulation results showed that on the premise of a reasonable solubility, a higher percentage of $S$ lead to a higher viscosity. Next, to determine the optimal composition of this copolymer, we tested its solubility. Results showed that when using diesel as a co-solvent, the optimal composition of this copolymer is 60\%PHFDA-24\%PSt-16\%S. Further experimental tests showed that this improved copolymer can increase the solution viscosity 100 -fold relative to neat carbon dioxide at $273 \mathrm{~K}$ and $28 \mathrm{Mpa}$ with a concentration of $1 \mathrm{wt} \%$.
\end{abstract}

Keywords: carbon dioxide, viscosity, dissipative particle dynamics, fracturing.

\section{Introduction}

Liquid carbon dioxide is widely used in two processes in the oil and gas industry; fracture stimulation and enhanced oil recovery $[1,2]$. Note that the viscosity of carbon dioxide is only $0.03-0.10 \mathrm{cp}$ at reservoir conditions. As the fracturing fluid, 
the effect of low fluid viscosity on proppant settling, placement of high sand concentration and fluid leakoff greatly reduces the effectiveness of fracture stimulation. And for enhanced oil recovery, the low viscosity of carbon dioxide causes it to finger towards the production wells and bypass large amounts of oil.

Many works have been done to thicken $\mathrm{CO}_{2}$ by using polymers [2-6]. These works are mainly divided into three basic strategies. The first strategy is by using extremely high molecular weight polymers. Unfortunately, although dense $\mathrm{CO}_{2}$ is a reasonably strong solvent for light hydrocarbons in crude oil, it is generally only a feeble solvent for higher molecular weight compounds. In the second strategy, relatively low molecular weight compounds capable of forming viscosityenhancing pseudo-networks of polymers via associations, hydrogen-bonding, or micelle formation were evaluated. These compounds contain polar groups that diminish carbon dioxide solubility. Hence, prohibitive amounts of co-solvent were needed to enhance these polymers' $\mathrm{CO}_{2}$ solubility for both strategies. The third group of studies included recent efforts to design novel viscosity enhancing molecules that exhibit very high carbon dioxide solubility. For example, Huang and coworkers [7] report that highly $\mathrm{CO}_{2}$-soluble fluorinated acrylate (PHFDA)/styrene (PSt)/ sulfonated styrene (S) copolymers can greatly enhance the viscosity of $\mathrm{CO}_{2}$. However, this copolymer are synthesized using expensive, highly fluorinated precursors, and a fraction of $5 \mathrm{wt} \%$ is needed to enhance the solution viscosity 180 -fold. As a result this copolymer still has to be improved to attain the desired level of viscosity when present in lower concentration. This requires a deep understanding of the link among micro molecular structures, their macroscopic rheological properties and the external shear flow.

As a mesoscale simulation algorithm, dissipative particle dynamics (DPD) is proposed and has successfully been used to investigate the phase behavior of various solution systems [8, 9]. Boek and Coveney [11] found this technique also a useful tool for studying the rheological properties of particulate suspensions $[10,11]$. They studied the flow of suspensions of solid spheres, rods and disks via applying a steady-shear rate, and found that the measured viscosities as a function of shear rate and volume fraction of the suspended particles were in excellent agreement with experiment results.

The same method was applied in this work to simulate the shear viscosity of surfactant solutions and their relationship with microstructure. Based on the simulated results, a new copolymer improved from Huang's work was synthesised. We further tested the rheological properties and showed the results in the last of this paper.

\section{Methodology and computation details}

In DPD, every bead represents a fluid element, which experiences other beads within a small cut-off radius. There are three types of forces in DPD algorithm, the random fore, dissipative force and conservative force, respectively. All beads are kept in motion by the random force and counteracted by the dissipative force depending on the velocity of surrounding beads. Together these counteracting forces act as a thermostat. The conservative forces between beads are determined 
by the conservative interaction parameters, which can be obtained from the FloryHuggins parameters. All forces are finite and smooth allowing for large time steps. For details on the algorithm we refer the reader to Groot and Warren [12].

In this work, PHFDA/PSt/S copolymers are simplified into simple DPD models. PHFDA beads, PSt beads and S beads are denoted by $\mathrm{H}, \mathrm{P}$ and $\mathrm{S}$, respectively, which are connected by springs. Carbon dioxide bead is denoted by $\mathrm{C}$ correspondingly. In order to optimize the molar ratio of this copolymer's monomers, nine different topological structures of this copolymer are investigated here, which are shown in table 1 . All these copolymers have the same degree of polymerization 20. Molar ratio of $\mathrm{H}$ beads is keeping on 60 percent to guarantee sufficient $\mathrm{CO}_{2}$-solubility, while fraction of $\mathrm{P}$ and $\mathrm{S}$ beads changes. The conservative interaction parameters are shown in table 2.

Table 1: Copolymers with different molar ratios of monomers investigated in the present work.

\begin{tabular}{|c|c|c|c|c|c|c|c|c|c|}
\hline $\begin{array}{c}\text { Copolymer } \\
\text { ID }\end{array}$ & 1 & 2 & 3 & 4 & 5 & 6 & 7 & 8 & 9 \\
\hline H (\%) & \multicolumn{10}{|c|}{60} \\
\hline P (\%) & 40 & 35 & 30 & 25 & 20 & 15 & 10 & 5 & 0 \\
\hline S (\%) & 0 & 5 & 10 & 15 & 20 & 25 & 30 & 35 & 40 \\
\hline
\end{tabular}

Table 2: Conservative interaction parameters $\mathrm{a}_{\mathrm{ij}}$.

\begin{tabular}{|c|c|c|c|c|}
\hline & C & H & P & S \\
\hline C & 25 & 15 & 26 & 78 \\
\hline H & 15 & 29 & 40 & 54 \\
\hline P & 26 & 40 & 25 & 39 \\
\hline S & 78 & 54 & 39 & 22 \\
\hline
\end{tabular}

A linear velocity profile at a fixed shear rate is imposed to these simulation systems, and the ratio of the resultant shear stress and the velocity profile gives the viscosity. In this work, the shear rate is fixed at 0.02 (DPD unit). However, problems arise at the boundaries of the simulation domain, as periodical boundary conditions are not able to maintain a steady linear velocity profile. To solve this problem, Lees-Edwards boundary conditions are applied here [13]. Note that viscous heating is absent here because of the built-in thermostat in DPD algorithm.

Dimensions of the simulation box are $L_{x}=20$ by $L_{y}=20$ by $L_{z}=20$, so the system contains 24,000 beads ( $\rho=3$ ). Volume fraction of the copolymer is fixed on $10 \%$. Firstly we run a simulation for 20,000 time steps with a time step of 0.01 to obtain the equilibrium state of these nine different copolymer systems under no shear. Then shear field is added to these systems to caculate the shear viscosity. The shear flow simulation runs 500,000 steps with a time step of 0.01 , with the last 100,000 steps to calculate the viscosity. 


\section{Results and discussion}

We first simulate these nine systems under no shear for 20,000 steps. Snapshots for parts of simulation systems are given in fig. 1. We can observe that for Copolymer 1, all molecules distribute randomly in solutions. There is no apparent bonding effect between different copolymer chains. However, for Copolymer 3, 5, 7 and 9, copolymer chains assemble via the bonding effect of S beads. For copolymers with higher fraction of $\mathrm{S}$ beads, copolymer molecules further form micells. It is mainly because of the attraction force between $\mathrm{S}$ beads.

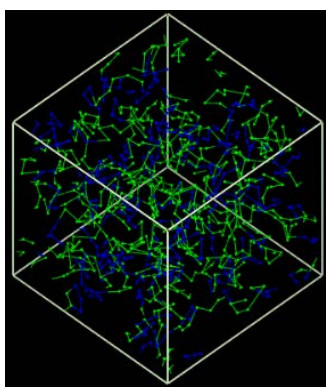

(a)

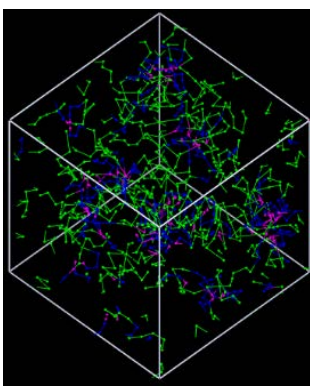

(b)

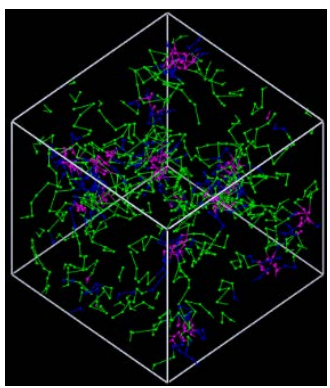

(c)

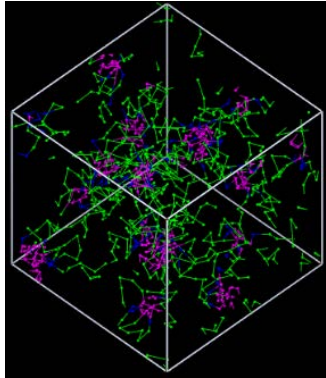

(d)

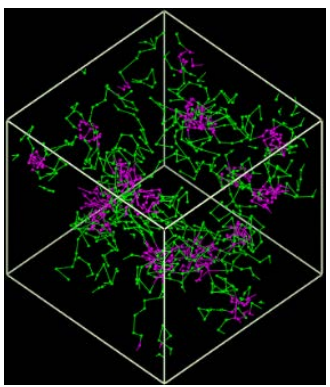

(e)

Figure 1: Snapshots of simulated polymer solution under no shear for Copolymer 1 (a), Copolymer 3 (b), Copolymer 5 (c), Copolymer 7(d) and Copolymer 9 (e). Here C beads are not shown for clarity, H, P and $\mathrm{S}$ particles are presented in green blue and purple, respectively.

After these systems reach steady state, a linear velocity profile is added to these systems. Thus we calculate the corresponding viscosities and show them in fig. 2. It is clearly seen that there is a strong positive correlation between molar ratio of $\mathrm{S}$ beads and the copolymer solution's viscosity. It is easy to understand: Sulfonated styrene (S bead) has a strong polar functional group $-\mathrm{SO}_{3}{ }^{2-}$, thus greatly enhance the bonding forces between different copolymer chains, leading to the enhancement of solution's viscosity. However, as S bead is insoluble in liquid $\mathrm{CO}_{2}$ a large fraction of $\mathrm{S}$ beads may greatly decreases the solubility of the copolymer. Thereby, we conclude that we should increase the sulfonation degree of this copolymer on the premise of a reasonable solubility. 


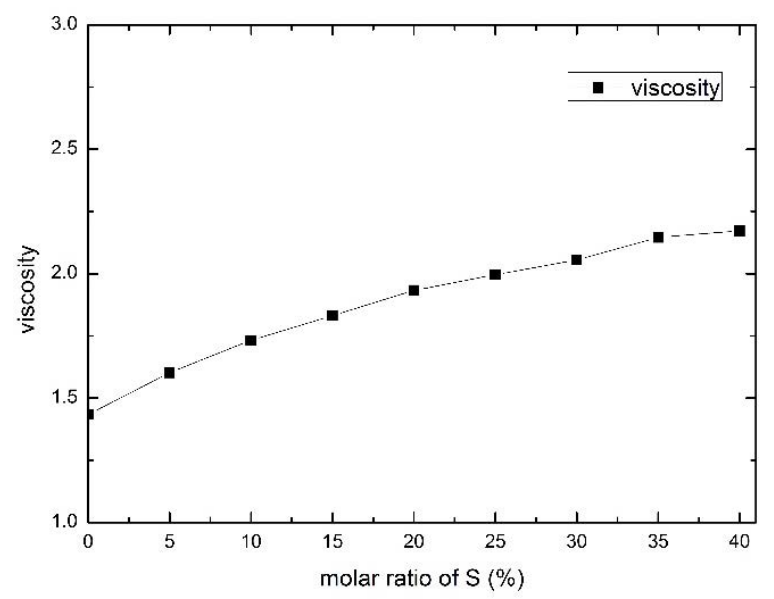

Figure 2: Calculated viscosity of solution as a function of S beads' molar ratio.

Using a further dissolving experiment, we finally fix the mole fraction of $\mathrm{H}, \mathrm{P}$ and $\mathrm{S}$ beads on $60 \%, 24 \%$ and $16 \%$, respectively. We name this new designed copolymer znj01. It is a very viscous fluid at room temperature and atmosphere pressure (fig. 3(a)), so we need some co-solvent to promote dispersion and solubility of znj01 in liquid carbon dioxide. It is found that znj01 can dissolve easily in diesel oil with a mass ratio of 1:2 (fig. 3(b)). Note that diesel also has high solubility in liquid carbon dioxide, so we choose diesel as the co-solvent. To test the effect of znj01 on thickening carbon dioxide, we use the Haake Mars III rotational rheometer, which has an excellent measuring accuracy for fluids with viscosity higher than $4 \mathrm{cp}$ (fig. 4). Viscosity of $1 \mathrm{wt} \%$ znj01, $2 \mathrm{wt} \%$ diesel oil and $97 \mathrm{wt} \%$ liquid carbon dioxide solution is then measured, results show that it can increase the solution viscosity 100 -fold relative to neat $\mathrm{CO}_{2}$ at $273 \mathrm{~K}$ and $28 \mathrm{MPa}$ with a concentration of $1 \mathrm{wt} \%$. The rheological curve is shown in fig. 5.

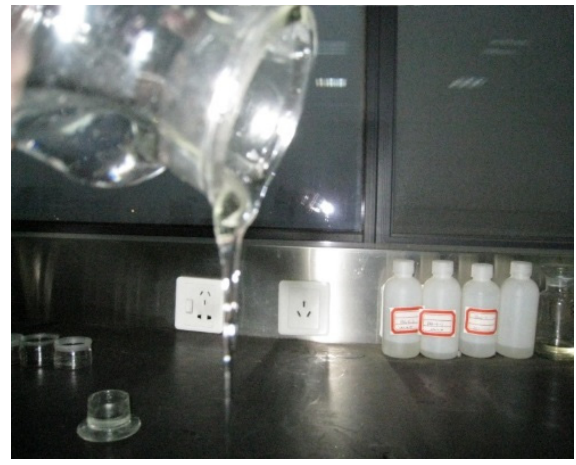

(a)

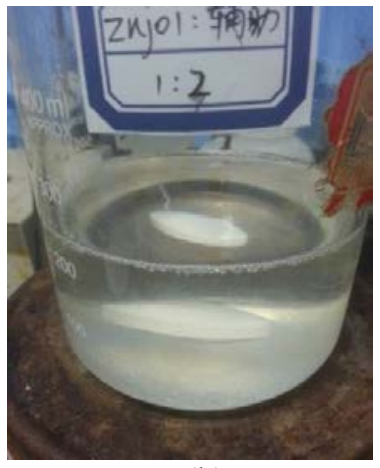

(b)

Figure 3: Pure znj01 (a) and its high solubility in diesel oil (b). 


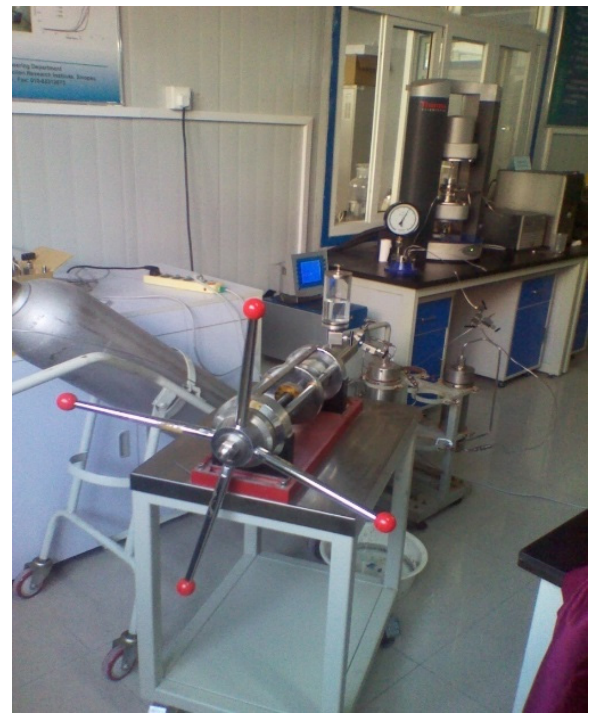

Figure 4: Hakke Mars III rotational rheometer system.

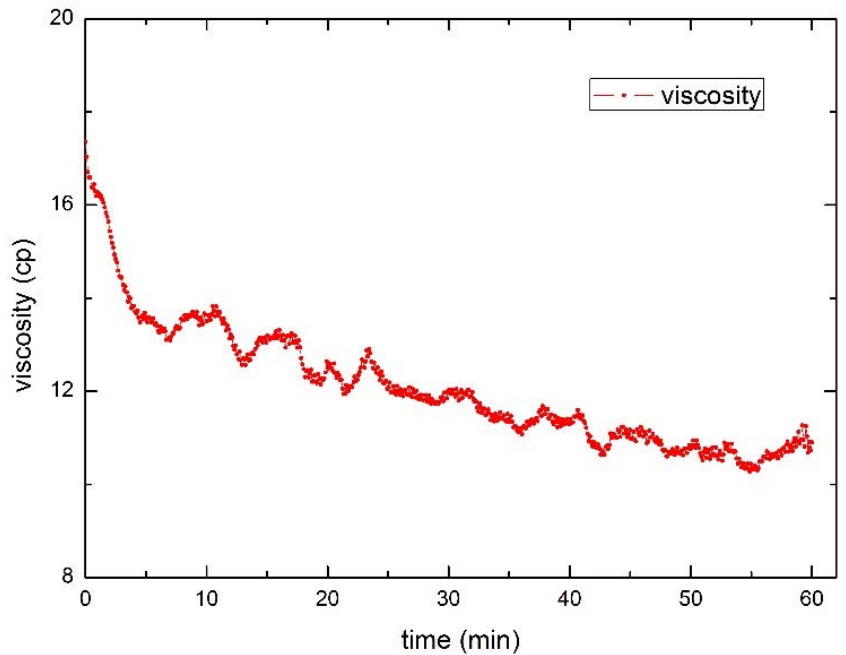

Figure 5: $\quad$ Rheological curve of $1 \mathrm{wt} \%$ znj01, 2wt\% diesel oil and 97wt\% liquid carbon dioxide solution system.

Up to now, znj01 has been applied to thicken liquid carbon dioxide in three times' liquid carbon dioxide fracturing constructions in China, including coal-bed gas wells and heavy oil wells. With a typical flow rate of $2-4 \mathrm{~m}^{3} / \mathrm{min}$, the sand ratio reaches around 5\% (fig. 6). 


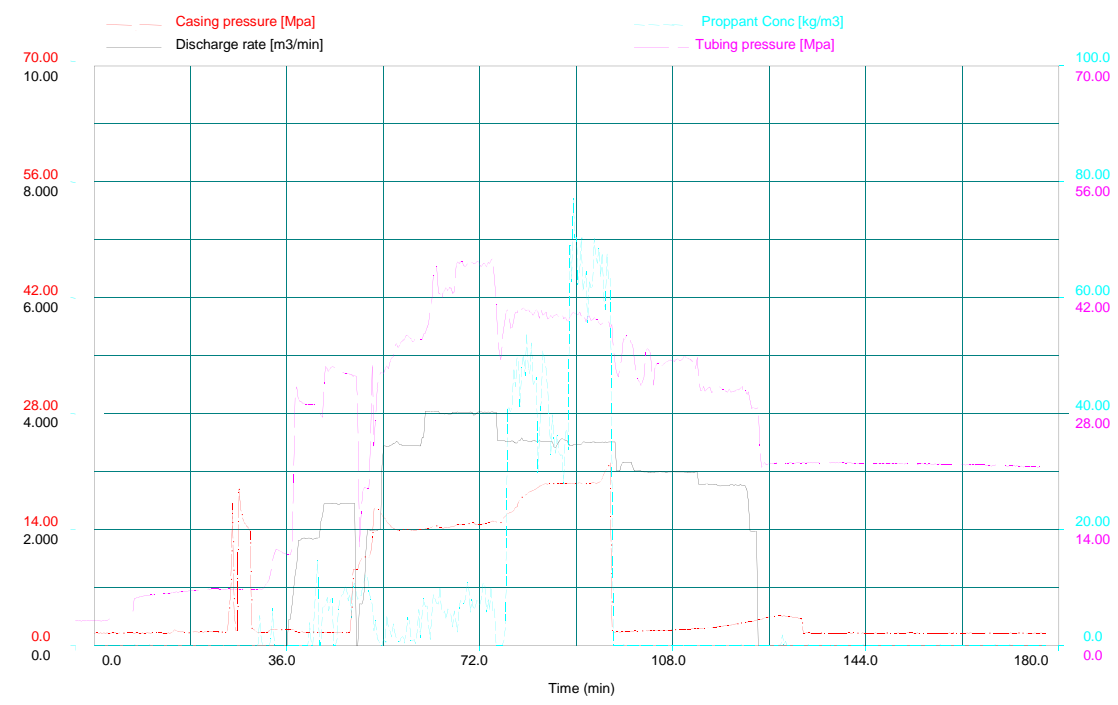

Figure 6: Construction curve of a liquid carbon dioxide fracturing using znj01.

\section{Conclusions}

We have performed DPD calculations to investigate the interactions between the topological structures of PHFDA/PSt/S copolymers and the viscosity of the copolymer solutions. Results show that the introduction of $S$ bead can enhance the attractive force between different copolymer chains, thus greatly thicken liquid carbon dioxide solutions. We conclude that we should increase the sulfonation degree on the premise of a reasonable solubility. Using a further dissolving experiment, we finally fix the mole fraction of $\mathrm{H}, \mathrm{P}$ and $\mathrm{S}$ beads on $60 \%, 20 \%$ and $20 \%$, respectively. Using diesel as co-solvent, the improved copolymers can increase the solution viscosity 100 -fold relative to neat CO2 at $273 \mathrm{~K}$ and $28 \mathrm{MPa}$ with a concentration of $1 \mathrm{wt} \%$.

\section{Acknowledgements}

This work is supported by Chinese Major national oil and gas projects 2008ZX05062 and Chinese National 973 project 2009CB219607.

\section{References}

[1] Liu H., Wang, F. \& Zhang J., et al., Fracturing with carbon dioxide: Application status and development trend. Petroleum exploration and development, 41(4), pp. 466-472, 2014. 
[2] Zhang, S.Y., She, Y.H. \& Gu, Y.A., Evaluation of polymers as direct thickeners for $\mathrm{CO}_{2}$ enhanced oil recovery. J. Chem. Eng. DATA, 56, pp. 1069-1079, 2011.

[3] Cummings, S., Trickett, K. \& Enick, R., et al., $\mathrm{CO}_{2}$ : a wild solvent, tamed. Phys. Chem. Chem. Phys., 13, pp. 1276-1289, 2011.

[4] Lan, H. Y. \& Tseng, H. C., Study on the rheological behavior of PP/supercritical $\mathrm{CO}_{2}$ mixture. J. Polym. Res., 9, pp. 157-162, 2002.

[5] Trickett, K., Xing, D. Z. \& Enick, R., et al., Rod-like micelles thicken $\mathrm{CO}_{2}$. Langmuir, 26(1), pp. 83-88, 2010.

[6] Shen, Z., McHugh, M. A. \& Xu, J., et al., $\mathrm{CO}_{2}$-solubility of oligomers and polymers that contain the carbonyl group. Polymer, 44, pp. 1491-1498, 2003.

[7] Huang, Z. H., Shi, C. M. \& Xu, J. H., et al., Enhancement of the viscosity of carbon dioxide using styrene/Fluoroacrylate copolymers. Macromolecules, 33, pp. 5437-5442, 2000.

[8] Meng, S. W., Zhang, J. \& Wu C., et al., Dissipative particle dynamics simulations of surfactant CTAB in ethanol/water mixture. Mol. Simulat., 40(13), pp. 1052-1058, 2014.

[9] Rekvig, L., Kranenburg, M. \& Vreede, J., et al., Investigation of surfactant efficiency using dissipative particle dynamics. Langmuir, 19, pp. 81958205, 2003.

[10] Koelman, J. M. V. A. \& Hoogerbrugge, P. J., Dynamic simulations of hardsphere suspensions under steady shear. Europhys. Lett., 21, pp. 363-368, 1993.

[11] Boek, E. S. \& Coveney, P. V., Simulating the rheology of dense colloidal suspensions using dissipative particle dynamics. Phys. Rev. E, 55, pp. 31243133, 1997.

[12] Groot, R. D. \& Warren, P. B., Dissipative particle dynamics: Bridging the gap between atomistic and mesoscopic simulation. J. Chem. Phys., 107, pp. 4423-4435, 1997.

[13] Lees, A. W. \& Edwards, S. F., The computer study of transport processes under extreme conditions. J. Phys. C., 5, pp. 1921-1928, 1972. 